\title{
Bowel dysfunction in young women with urinary retention
}

\author{
M-C Lemieux, M A Kamm, C J Fowler
}

\begin{abstract}
Urinary retention in young women is rare, but a syndrome has recently been described in which urinary retention is associated with abnormal periurethral electromyography. The incidence of bowel dysfunction in these women was investigated, in an attempt to determine whether this might be a more widespread disorder. Of 12 patients with this urological abnormality, eight were constipated. No consistent anorectal abnormality was identified on anorectal physiological testing. The electromyographic abnormality was not seen in the external anal sphincter. One patient had an abnormality of the internal anal sphincter smooth muscle, while another had a generalised disorder of the gastrointestinal tract and urinary bladder resembling a visceral myopathy. Bowel symptoms are common in this group of women with urinary retention, but abnormalities of bowel function are not specific. A common mechanism for bladder and bowel symptoms remains a possibility. (Gut 1993; 34: 1397-1399)
\end{abstract}

Until recently, in the absence of overt neurological disease, the cause of urinary retention in most young women was unknown or believed to be psychological in origin. A syndrome has recently been described, however, in which the striated urethral sphincter shows abnormal electromyographic activity characterised by decelerating bursts and complex repetitive discharges. ${ }^{12}$ This electrical activity is thought to cause failure of relaxation of the sphincter while attempting to void. This outflow obstruction is different from detrusor sphincter dyssynergia in that the electromyographic abnormality can be recorded at rest and is qualitatively different. Complex repetitive discharges have also been seen in other urinary dysfunctions such as stress incontinence, where the activity was called pseudomyotonia. ${ }^{3}$ An association between the abnormal spontaneous electromyographic activity and the polycystic ovary syndrome has been described. ${ }^{2}$

In view of the shared innervation of the pubococcygeus muscle, external anal sphincter and urethral striated sphincter, we wished to discover if this urethral sphincter abnormality was an isolated phenomenon or whether it affected other pelvic structures. It had also come to our attention that a seemingly disproportionate number of these women complained of bowel symptoms.

Methods and patients

Our records were examined to identify a cohort of women who had originally presented to other hospitals with acute urinary retention of unknown cause. It was possible to contact 12 of 21 patients identified. The average age was 30 years (range $18-47$ years).

All had been referred for specialist electromyographic evaluation. Urethral electromyography was performed using the transperineal approach and a concentric needle electrode. All were found to have the characteristic electromyographic abnormality of decelerating bursts and complex repetitive discharges.

A detailed questionnaire was used to assess the symptoms of the gastrointestinal and genitourinary tract. Patients were asked about their bowel frequency, the need to strain at stool, and the need for laxatives. The time of onset of bowel symptoms was noted, and in particular the relation to the onset of urinary symptoms. Al patients were interviewed by one investigator (MCL).

When patients complained of any bowel symptoms they were offered further physiological investigation of the anorectum. This included measurement of the maximum resting and voluntary contraction anal pressures with a microballoon system. ${ }^{5}$ The first is a measure of the function of the smooth muscle internal anal sphincter and the second is a measure of the function of the striated external anal and puborectalis muscles. Rectal sensation to balloon distension was assessed using an intrarectal balloon distended with air. ${ }^{6}$ The volumes required to elicit an initial sensation, sensation of urgency, and maximum tolerated volumes were all noted. Rectal and anal sensation was also assessed using an electrical stimulus. ${ }^{7}$ Pudendal nerve function was assessed by measuring its latency in the distal portion between the ischial spine and the external anal sphincter. ${ }^{8}$

Anorectal manometry was compared with two control groups. The first was a group of 13 healthy age matched women (mean age 31 , range 23-47) without bowel symptoms. The second was a group of 20 age matched women (mean age 32 , range 18-44) with severe idiopathic constipation and no urinary symptoms.

Electromyography of the external anal sphincter was performed using a concentric needle electrode to determine if the electromyographic abnormality seen in the urethral sphincter was also present in this muscle.

\section{Results}

At the time of interview all women were still experiencing voiding dysfunction. Six $(50 \%)$ were requiring intermittent self catheterisation, in a frequency varying from once to six times daily. 
Using Drossman's definition of constipation (straining more than 25 per cent of the time or a bowel frequency less than twice per week) ${ }^{9}$ eight of the 12 women were constipated. The other four patients denied any defecatory symptoms. Seven patients agreed to have their anorectal function evaluated.

The mean (SD) resting anal pressure was 120 (30) $\mathrm{cm}$ of water. This was significantly greater than the healthy control group $\left(120\right.$ (12) $\mathrm{cm} \mathrm{H}_{20}$ $v 90$ (10), mean (SEM), patients $v$ healthy controls, $\mathrm{p}=0.03)$. When compared with the control group of constipated women without urinary symptoms there was no significant difference in their mean maximum resting anal pressures $(p=0 \cdot 19)$.

Two patients had appreciably raised maximum resting anal pressures. One had marked ultra slow wave activity with a maximum pressure of $180 \mathrm{~cm} \mathrm{H} \mathrm{H}_{2} \mathrm{O}$ (normal upper limit for our laboratory is $100 \mathrm{~cm} \mathrm{H}_{2} \mathrm{O}$ ), while in another patient the maximum resting anal pressure was $140 \mathrm{~cm} \mathrm{H}_{2} \mathrm{O}$.

The sensory response to intrarectal balloon distension was normal in all but two patients, and anal or rectal mucosal sensitivity was also abnormal in two other patients. Only one patient tested had prolonged pudendal nerve terminal motor latencies.

External anal sphincter electromyography in four patients did not show any abnormal activity of that muscle, by contrast with another study where seven of eight women with complex repetitive discharges in their urethral sphincter also had evidence of similar activity in their striated anal sphincter. ${ }^{10}$

\section{Case report}

An 18 year old nulliparous woman had been well until a trip abroad 18 months previously. Upon her return she suffered from a flu like illness after which she became constipated, requiring oral laxatives as well as repeated admissions to hospital for enemas. Bowel actions during this period were four weeks apart despite laxatives. Simultaneous with the constipation she developed abdominal bloating and vomiting after meals. Two months before referral she started voiding infrequently.

A contrast study of the large bowel showed a dilated impacted rectum. The colonic transit time was considerably prolonged when studied using radio-opaque markers. Radioisotopic studies of oesophageal and gastric transit showed appreciable delay in both. Urodynamic investigations showed a large atonic bladder with incomplete emptying (bladder capacity $917 \mathrm{ml}$ with a post-void residual volume of $407 \mathrm{ml}$ and a flow rate of $16 \mathrm{ml} / \mathrm{sec}$ ). Urethral sphincter electromyography showed abnormal declerating bursts.

A full thickness rectal biopsy specimen showed normal ganglion distribution but the circular layer of the muscularis mucosa showed an unusual degree of splitting of muscle bundles by interstitial connective tissue. Nerve stains (S100) showed abnormal nerve fibres extending from the myenteric plexus into both layers of the muscularis propria; these changes were abnormal, and felt to be consistent with a diagnosis of chronic intestinal pseudo-obstruction, but it could not be discovered if this was a primary myopathic or neuropathic disorder.

The upper gastrointestinal symptoms have partly responded to treatment with oral cisapride, but she still requires enemas and urinary self catheterisation.

\section{Discussion}

The main finding in this study is a high prevalence of bowel symptoms in young women with 'idiopathic' urinary retention. Although constipation is common in women, the proportion of affected women in this group of patients is higher than one would expect to find in the general population. ${ }^{9}$

The resting anal pressure in these women was raised, but this was not different from a control group of constipated women. It is therefore not clear whether this is a non-specific finding, or one related to the urinary disorder.

Previous studies have shown a high incidence of a variety of urological symptoms in constipated women, including hesitancy, urgency, changed frequency, and stress incontinence..$^{112}$ A variety of urodynamically proved diagnoses have been identified in women with idiopathic constipation, including detrusor sphincter dyssynergia, acontractile bladder, detrusor instability, stress incontinence, and obstructed urinary flow. ${ }^{13}$ One of the commonest findings has been an increased bladder capacity. ${ }^{14}$ The association of constipation, however, with overt urinary retention and a reported electromyographic abnormality has not been described.

A previous study found that in 10 patients presenting with difficult rectal evacuation due to pelvic floor incoordination, urinary flow was slow and bladder capacity was increased compared with a healthy control group. ${ }^{13}$ No patient was in urinary retention, however, and no urethral electromyographic studies were therefore performed.

Patients with a well documented visceral myopathy affecting intestinal smooth muscle are also known to have involvement of their urinary tract sometimes. This diagnosis is usually clear and clinically distinguishable from the patients presented in this report (apart from the case report described).

Two of the patients in this series deserve special attention. In one patient there was objective evidence of a neuromuscular visceral disorder affecting the gastrointestinal tract. Another patient with an abnormally high resting anal pressure and considerable ultra slow wave activity in the anal sphincter seemed to have an abnormality of anal sphincter smooth muscle. Both patients had urinary retention with characteristic electromyographic findings recorded from the striated urethral sphincter muscle. How could the involvement of different types of muscle have occurred? It may be that the complete urinary retention might not have occurred if there had not also been an abnormality of the bladder smooth muscle. This abnormality may be a shared smooth muscle disorder between the bladder and the bowel. 
Bowel symptoms are common in this unusual group of women with idiopathic urinary retention and specific periurethral electromyographic findings. Our study has shown that the striated muscle electromyographic abnormality does not affect the anal sphincter to account for this phenomenon of urinary retention and constipation.

MCL was a MacLaughlin Fellow (Canada). MAK was supported by the St Mark's Research Foundation.

1 Fowler CJ, Kirby RS. Abnormal electromyographic activity (decelerating bursts and complex repetitive discharges) in the striated muscle of the urethral sphincter in 5 women with persisting urinary retention. BrF Urol 1985; 57: 67-70.

2 Fowler CJ, Christmas TJ, Chapple RC, Fitzmaurice Parkhouse H, Kirby RS, Jacobs HS. Abnormal electromyographic activity of the urethral sphincter, voiding dysfunction, and polycystic ovaries: A new syndrome? $B M \mathcal{T}$ 1988; 297: 1436-8.

3 Butler WJ. Pseudomyotonia of the periurethral sphincter in women with urinary incontinence. $\mathcal{F}$ Urol 1979; 122: 838-40.

4 Fowler $\mathrm{CJ}$, et al. Individual motor unit analysis in the diagnosis of disorders of urethral sphincter innervation. 7 Neurol Neurosurg Psychiatry 1984; 47: 637-41.

5 Neill ME, Parks AG, Swash M. Physiological studies of the anal sphincter musculature in faecal incontinence and recta prolapse. BrF Surg 1981; 68: 531-6.

6 Ihre T. Studies on anal function in continent and incontinen patients. Scand F Gastroenterol 1974; 9 (suppl): 1-64.

7 Kamm MA, Lennard-Jones JE. Rectal mucosal electrosensory testing - Evidence for a rectal sensory neuropathy in idiopathic constipation. Dis Colon Rectum 1990; 33: 419-23.

8 Kiff ES, Swash M Slowed conduction in the pudendal nerves in idiopathic (neurogenic) faecal incontinence. $\mathrm{Br} \mathcal{F}$ Surg 1984; 71: 614-6.

9 Drossman DA. Bowel patterns among subjects not seeking health care. Gastroenterology 1982; 83: 529-34.

10 Webb RJ, Fawcett PRW, Neal DE. Electromyographic abnormalities in the urethral and anal sphincters of women

11 Bannister JJ, Lawrence WT, Smith A, Thomas DG, Read $\mathrm{NW}$. Urological abnormalities in young women with severe constipation. Gut 1988; 29: 17-20.

12 Preston DM, Lennard-Jones JE. Severe chronic constipation of young women: idiopathic slow transit constipation. $G u$ 1986; 27: 41-8.

13 MacDonald A, Shearer M, Paterson PJ, Finlay IG. Relation ship between outlet obstruction constipation and obstructed urinary flow. Brf Surg 1991; 78: 693-5.

14 Kerrigan DD, Lucas MG Sun WM, et al. Idiopathic constipation associated with impaired urethrovesical and sacral pation associated with impaired u; 4 rethrovesic

15 Milla P. Chronic intestinal pseudo-obstruction. In: Kamm MA, Lennard-Jones JE, eds. Gastrointestinal transit. Pathophysiology and pharmacology. Petersfield: Wrightson Biomedical Publishing, 1991: 157-68. 